\title{
CSF3R-mutated chronic neutrophilic leukemia: long-term outcome in 19 consecutive patients and risk model for survival
}

\author{
Natasha Szuber ${ }^{1}$, Christy M. Finke', Terra L. Lasho ${ }^{1}$, Michelle A. Elliott ${ }^{1}$, Curtis A. Hanson ${ }^{2}$, Animesh Pardanani ${ }^{1}$ and \\ Ayalew Tefferi $^{1}$
}

Chronic neutrophilic leukemia $(\mathrm{CNL})$ is a rare $B C R$ $A B L$ negative myeloproliferative neoplasm (MPN), whose molecular pathogenesis transitioned from obscurity to the limelight with the seminal identification of oncogenic colony stimulating factor 3 receptor (CSF3R) mutations in the vast majority of CNL patients in $2013^{1}$. The unifying features of CNL consist of sustained mature neutrophil proliferation, bone marrow granulocytic hyperplasia, and hepatosplenomegaly, and while clinical manifestations and disease course remain heterogeneous, prognosis is often unfavorable ${ }^{2}$. Due to both the historically challenging diagnostic confirmation and the rarity of CNL, comprehensive analyses of World Health Organization (WHO)-defined, molecularly annotated populations of CNL patients have been scarce and often limited by small cohorts. Although the more common CSF3RT618I is perceived as the "hallmark" genetic lesion, there is little information on how individuals harboring other CSF3R mutations may be distinguished from their CSF3RT618Imutated counterparts. Furthermore, while there is some evidence that variables such as high leukocyte count ${ }^{3}$, thrombocytopenia, and the presence $A S X L 1$ mutations may define a higher-risk subset of CNL patients ${ }^{2}$, there are limited data on prognostic factors and long-term survival in CNL and consequently, no existing operational risk model or prognostic scoring system. The objective of this study was to determine the characteristics, treatment patterns, and long-term overall survival (OS) in 19

\footnotetext{
Correspondence: Ayalew Tefferi (tefferi.ayalew@mayo.edu)

'Division of Hematology, Mayo Clinic, Rochester, MN, USA

Division of Hematopathology, Department of Laboratory Medicine, Mayo Clinic, Rochester, MN, USA
}

consecutive WHO-defined CSF3R-mutated CNL patients - the largest cohort to date. Furthermore, we sought to identify and integrate adverse prognostic factors into a risk model predictive of inferior overall survival in CNL.

Nineteen consecutive patients evaluated at the Mayo Clinic harboring CSF3R mutations and meeting WHO criteria for $\mathrm{CNL}^{4}$ were retrospectively identified. Details of clinical characteristics, laboratory parameters including molecular and cytogenetics data, treatment regimens and responses, as well as disease evolution were carefully abstracted from medical records. Diagnosis was confirmed in all cases by review of peripheral blood counts and smears, and bone marrow (BM) aspirates and biopsies. Mutation analysis of CSF $3 R, A S X L 1$, and SETBP1 was conducted as previously described ${ }^{5,6}$. Analyses were based on clinical and laboratory parameters obtained at diagnosis. OS was calculated as an interval from the time of diagnosis to last follow-up or death. Survival analysis was performed by the Kaplan-Meier method and differences assessed using the log-rank test. Conventional statistical methods were used for all analyses. Statistical analyses were performed using Stat View software (SAS Institute, Cary, NC, USA).

From October 1995 to October 2017, 19 consecutive WHO-defined CSF3R-mutated patients were evaluated at the Mayo Clinic. Detailed clinical and laboratory characteristics of this cohort are presented in Table 1. Mutations in CSF3R included the classical T618I mutation in 14 patients and other mutations including M696T in 2 patients and $\mathrm{T} 640 \mathrm{~N}$, c. $2215 \mathrm{C}>\mathrm{T}$ truncation mutation, and I598I SYN in one patient each. Median age was 68 (range 26-87 years) and there was a slight preponderance 
Table 1 Characteristics and outcomes in chronic neutrophilic leukemia patients with CSF3RT618I mutations versus other CSF3R variants $(n=19)$

\begin{tabular}{|c|c|c|c|c|}
\hline Characteristics & $\begin{array}{l}\text { All patients } \\
(n=19)\end{array}$ & $\begin{array}{l}\text { CSF3RT618I-mutated } \\
(n=14)\end{array}$ & $\begin{array}{l}\text { Other CSF3R mutations }{ }^{a} \\
(n=5)\end{array}$ & $p$-value \\
\hline Median age at diagnosis (years) & 68 & 74 & 59 & 0.09 \\
\hline Gender, male (\%) & $11(58 \%)$ & $9(64 \%)$ & $2(40 \%)$ & 0.35 \\
\hline Prior cytotoxic exposure & $4 / 19(21 \%)$ & $3 / 14(21 \%)$ & $1 / 5(20 \%)$ & 0.95 \\
\hline Family history hematological neoplasm & $4 / 19(21 \%)$ & $3 / 14(21 \%)$ & $1 / 5(20 \%)$ & 0.95 \\
\hline History of thrombosis & 8/18 (44\%) & $7 / 13(54 \%)$ & $1 / 5(20 \%)$ & 0.20 \\
\hline History of hemorrhage & $5 / 18(28 \%)$ & $5 / 13(39 \%)$ & $0 / 5(0 \%)$ & 0.10 \\
\hline Presence of palpable splenomegaly (\%) & $9 / 18(50 \%)$ & $7 / 13(54 \%)$ & $2 / 5(40 \%)$ & 0.60 \\
\hline Leukocyte count $\left(\times 10^{9} / \mathrm{L}\right)$ & 65.9 & 76.4 & 34.0 & 0.40 \\
\hline Leukocytes $>60 \times 10^{9} / \mathrm{L}^{b}$ & $10 / 19(53 \%)$ & $9 / 14(64 \%)$ & $1 / 5(20 \%)$ & 0.09 \\
\hline Hemoglobin (g/dL) & 12.0 & 9.6 & 13.6 & 0.06 \\
\hline Hemoglobin $<10 \mathrm{~g} / \mathrm{dL}$ & $9 / 19(47 \%)$ & $8 / 14(57 \%)$ & $1 / 5(20 \%)$ & 0.15 \\
\hline Platelet count $\left(\times 10^{9} / \mathrm{L}\right)$ & 227 & 158 & 299 & 0.04 \\
\hline Platelets $<160 \times 10^{9} / L^{b}$ & $8 / 19(42 \%)$ & $8 / 14(57 \%)$ & $0 / 5(0 \%)$ & 0.03 \\
\hline Metamyelocytes + myelocytes (\%) & $2 \%$ & $2 \%$ & $2 \%$ & 0.82 \\
\hline Monocytes (\%) & $2 \%$ & $1 \%$ & $5 \%$ & 0.11 \\
\hline LDH (U/L) & 234 & 234 & 234 & 0.50 \\
\hline Abnormal karyotype (\%) & $2 / 19(11 \%)$ & $2 / 14(14 \%)$ & $0 / 5(0 \%)$ & 0.37 \\
\hline SETBP1 mutation (\%) & $6 / 19(32 \%)$ & $5 / 14(36 \%)$ & $1 / 5(20 \%)$ & 0.52 \\
\hline ASXL1 mutation (\%) & 9/19 (47\%) & $6 / 14(43 \%)$ & $3 / 5(60 \%)$ & 0.51 \\
\hline \multicolumn{5}{|l|}{ Therapy regimens } \\
\hline \multicolumn{5}{|l|}{ First line therapy } \\
\hline Hydroxyurea & $14 / 17$ (82\%) & $9 / 12(75 \%)$ & $5 / 5(100 \%)$ & NA \\
\hline Interferon-alpha & $1 / 17(6 \%)$ & $1 / 12(8 \%)$ & - & \\
\hline Other agent ${ }^{c}$ & $2 / 17(12 \%)$ & $2 / 12(17 \%)$ & - & \\
\hline \multicolumn{5}{|l|}{ Second line therapy } \\
\hline Ruxolitinib & $3 / 10(30 \%)$ & $2 / 8(25 \%)$ & $1 / 2(50 \%)$ & \\
\hline Stem cell transplant & $1 / 10(10 \%)$ & $1 / 8(13 \%)$ & - & \\
\hline Other agent ${ }^{c}$ & $6 / 10(60 \%)$ & $5 / 8(62 \%)$ & $1 / 2(50 \%)$ & \\
\hline \multicolumn{5}{|l|}{ Third line therapy } \\
\hline Stem cell transplant & $1 / 6(17 \%)$ & - & $1 / 2(50 \%)$ & \\
\hline Ruxolitinib & $1 / 6(17 \%)$ & $1 / 4(25 \%)$ & - & \\
\hline Other agent (Cladribine or Decitabine) & $2 / 6(33 \%)$ & $1 / 4(25 \%)$ & $1 / 2(50 \%)$ & \\
\hline Combination therapy $^{d}$ & $2 / 6(33 \%)$ & $2 / 4(50 \%)$ & - & \\
\hline Requiring 2nd line therapy & $10(53 \%)$ & $8(57 \%)$ & $2(40 \%)$ & 0.51 \\
\hline Requiring 3rd line therapy or more & $6(32 \%)$ & $4(29 \%)$ & $2(40 \%)$ & 0.64 \\
\hline Status last follow-up, dead (\%) & $10(53 \%)$ & $8(57 \%)$ & $2(40 \%)$ & 0.51 \\
\hline \multicolumn{5}{|l|}{ Evolution } \\
\hline AML (\%) & $3 / 19(16 \%)$ & $2 / 14(14 \%)$ & $1 / 5(20 \%)$ & 0.76 \\
\hline
\end{tabular}


Table 1 continued

\begin{tabular}{|c|c|c|c|c|}
\hline Characteristics & $\begin{array}{l}\text { All patients } \\
(n=19)\end{array}$ & $\begin{array}{l}\text { CSF3RT6181-mutated } \\
(n=14)\end{array}$ & $\begin{array}{l}\text { Other CSF3R mutations }{ }^{a} \\
(n=5)\end{array}$ & $p$-value \\
\hline CMML (\%) & $2 / 19(11 \%)$ & $1 / 14(7 \%)$ & $1 / 5(20 \%)$ & 0.42 \\
\hline Time to last follow-up or death & 22.4 months & 17.2 months & 42.7 months & 0.03 \\
\hline
\end{tabular}

Bold indicate statistically significant values

$A M L$ acute myeloid leukemia, $C M M L$ chronic myelomonocytic leukemia, $L D H$ lactate dehydrogenase, NA not available

${ }^{a}$ Other CSF3R mutations: M696T $(n=2)$, T640N, c.2215C $>$ T truncation mutation, and I598I SYN ( $n=1$ each)

beukocyte count $>60$ and platelet count $<160 \times 10^{9} / \mathrm{L}$ cut-off values determined by ROC analysis

'Other therapy regimens: thalidomide, tyrosine kinase inhibitors (imatinib, dasatinib), cladribine, azacitidine, and hydroxyurea plus thalidomide

${ }^{\mathrm{d} C}$ Combination therapies included hydroxyurea plus nilotinib in one patient and splenectomy followed peri-operatively by hydroxyurea plus interferon-alpha in another

of males (58\%). Overall, few patients had prior cytotoxic exposure or a family history of hematological neoplasm (20-21\% each). When stratified according to the presence of CSF3RT618I versus other CSF3R mutations, those carrying T618I were older (median age 74 versus 59 years), showed greater male predominance (64 versus $40 \%$ male), had a more frequent history of thrombosis (54 versus $20 \%$ ) and hemorrhage (39 versus $0 \%$ ), and more commonly presented palpable splenomegaly (54 versus $40 \%$ ) and an abnormal karyotype (14 versus $0 \%$ ) at diagnosis, though these did not achieve statistical significance. Further, those harboring CSF3RT618I presented a trend towards higher leukocyte counts $\left(76.4\right.$ versus $\left.34 \times 10^{9} / \mathrm{L}\right)$ and lower hemoglobin values ( 9.6 versus $13.6 \mathrm{~g} / \mathrm{dL}$ ) as well as significantly lower platelet counts (158 versus $299 \times 10^{9} / \mathrm{L} ; p=0.04$ ) compared to individuals carrying other CSF3R mutations. The mutational frequencies of SETBP1 and ASXL1 mutations respectively were: overall 32 and 47\%; in CSF3RT618I-mutated individuals 36 and 43\%; and in those with other CSF3R mutations 20 and $60 \%$, though differences between the two groups were not statistically significant. Two of fourteen CSF3RT618Imutated (14\%) and one of five patients with other CSF3R mutations (20\%) evolved to acute myeloid leukemia (AML) while one of fourteen (7\%) and one of five (20\%) respectively evolved to chronic myelomonocytic leukemia (CMML). Median time to last follow-up or death was 22.4 months and was significantly shorter in CSF3RT618I-mutated patients compared to those carrying other CSF3R mutations (17.2 versus 42.7 months; $p=$ 0.03).

Using a receiver operating characteristics (ROC) analysis, optimal cut-off points for defining low/high-risk disease were determined for leukocyte and platelet counts. On univariate analysis, platelet count below $160 \times 10^{9} / \mathrm{L}$, leukocyte count above $60 \times 10^{9} / \mathrm{L}$, and presence of an ASXL1 mutation were associated with significantly inferior OS $(p<0.05)$ and all three maintained significance on multivariate analysis with respective $p$-values of 0.001 ,
0.036, and 0.016. Based on these parameters, weighted risk points were attributed commensurate with the risk ratio (RR) of each variable to define a scoring system predictive of CNL patient survival: platelets below $160 \times$ $10^{9} / \mathrm{L}\left(\mathrm{RR}=16 ; 2\right.$ points), leukocytes above $60 \times 10^{9} / \mathrm{L}$ $(\mathrm{RR}=5,1$ point), and presence of $A S X L 1$ mutation ( $\mathrm{RR}=$ 6,1 point). Using this risk model, patients were assigned a low-risk (0-1 points; $n=9)$ or high-risk (2-4 points; $n=$ 10) designation and risk-stratified Kaplan-Meier survival curves confirmed significantly decreased OS in high-risk patients (median OS 22.4 months versus not yet reached; log rank $p=0.0016$ ) (Fig. 1).

Treatment regimens and responses are presented in Table 1 . The majority of patients were treated with hydroxyurea as a first-line agent (82\%) and it was ultimately received by all $(100 \%)$ at some point over their disease course. Most patients (53\%) required second-line therapy and nearly a third (32\%) required three lines of treatment or more. In this cohort, four patients, all having previously been exposed to hydroxyurea, received treatment with JAK inhibitor ruxolitinib. In three instances, ruxolitinib was administered as a second-line therapy and in one case, as a third-line agent. Reponses to ruxolitinib were varied: in one case, treatment was ongoing with favorable response but had been initiated recently ( $\sim 2$ months prior to data collection), in two other cases there was an initial response but eventual worsening of leukocytosis requiring subsequent additional therapies (duration of responses $\sim 9.5$ and 36 months, respectively), and in one case, ruxolitinib was received during blast phase disease as a "bridge" to transplant for a duration of only $\sim 0.5$ months; this patient ultimately had a favorable outcome and was alive at last follow-up, approximately 46 months from initial diagnosis. Two patients ultimately underwent hematopoietic stem cell transplant (HSCT), both 3 months following blast transformation; while one patient succumbed to complications of HSCT (venoocclusive disease and ultimately death secondary to infection), the other (patient having received ruxolitinib 


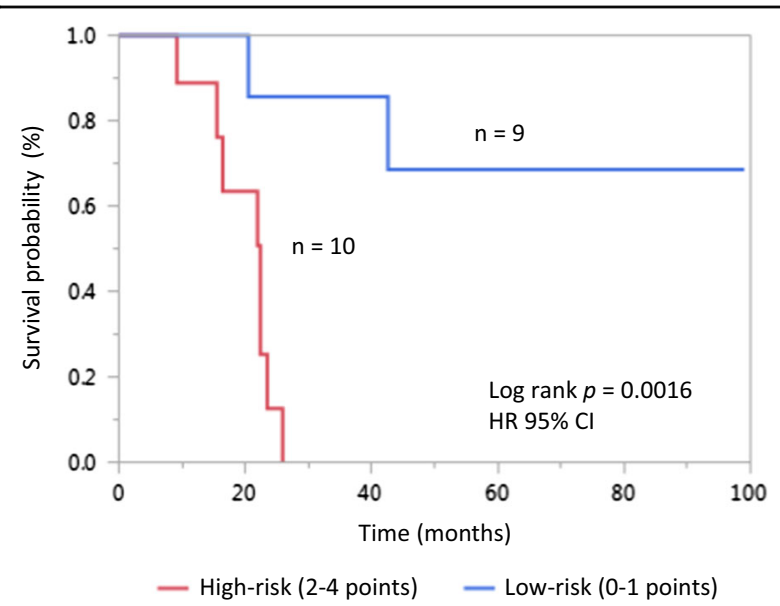

Fig. 1 Risk-stratified Kaplan-Meier survival curves for 19 CSF3Rmutated CNL patients. Points were attributed for ROC analysisdefined cut-offs of platelet count $<160 \times 10^{9} / \mathrm{L}$ (2 points), leukocyte count $>60 \times 10^{9} / \mathrm{L}$ (1 point) and presence of ASXL1 mutation (1 point) according to their respective risks ratios and patients stratified into low-risk (0-1 points) or high-risk (2-4 points) groups using this prognostic risk model (log rank $p=0.0016)$

pre-HSCT) experienced a favorable outcome and showed no signs of relapse $\sim 40$ months post-transplant.

As the largest-scale report of consecutive CSF3Rmutated CNL patients to date, this study yielded several novel and clinically useful findings. First, the assessment of long-term outcomes confirmed the generally aggressive course of CNL with a median survival of less than 2 years, consistent with historical data ${ }^{7}$, and a requirement for two or more lines of therapy in most patients. Although only $\sim 16 \%$ of patients formally evolved to AML, most patients eventually experienced a worsening of leukocytosis and an increased requirement for transfusions, regardless of therapeutic agent, and blast transformation was eventually suspected in a number of additional patients, though unconfirmed.

Second, by subdividing CSF3R-mutated CNL patients into mutational subgroups, specifically T618I versus other CSF3R mutations, we identified two phenotypically and prognostically distinct subsets of CNL patients. CSF3RT618I-mutated individuals cluster with adverse clinical characteristics, represent a prognostically less favorable group overall and likely correspond to the definitive molecularly-defined CNL entity. Further studies explicitly appraising the less prevalent, diverselycomposed molecular subset of "other" CSF3R mutations will be required to validate these findings. Interestingly, the question of whether these non-T618I CSF3R mutations should still be considered accurate molecular markers of $\mathrm{CNL}$ is complex, requires further investigation, and cannot be addressed within the confines of this communication.

Importantly, we integrated the three variables predictive of inferior survival on multivariate analysis weighted for risk to develop low-risk and high-risk patient categories and an operational risk model for survival in CNL (platelet count $<160 \times 10^{9} / \mathrm{L}=2$ points, leukocyte count $>60 \times 10^{9} / \mathrm{L}=1$ point, and presence of $A S X L 1$ mutation $=1$ point; low-risk $0-1$ points, high-risk $2-4$ points). This is the first prognostic scoring system to be reported for risk assessment in CNL. From a practical standpoint, we propose that individuals classified as high-risk be counseled regarding the prognostic implications of their disease, undergo closer monitoring for signs heralding disease transformation and perhaps even be considered earlier on for more intensive therapeutic approaches such as hematopoietic stem cell transplant.

Finally, although interesting preliminary data exist ${ }^{6,8-10}$, there has been limited clinical experience using ruxolitinib in patients with CNL. Ruxolitinib was received by four patients in our cohort with varying responses. In all cases, it was successful in initially controlling leukocyte count and in one instance of application pre-HSCT, its potential contribution to a favorable post-transplant outcome remains uncertain. However, in $50 \%$ of the cases, there was an eventual, albeit variably-timed loss of response, suggesting that ruxolitinib has limited if any disease-modifying effect. Thus, while it may be justified to maintain this agent in our arsenal of CNL-directed therapy, identifying effective treatments capable of inducing durable remission are clearly an unmet need in this disease.

\section{Conflict of interest}

The authors declare that they have no conflict of interest.

\section{Publisher's note}

Springer Nature remains neutral with regard to jurisdictional claims in published maps and institutional affiliations.

Received: 3 January 2018 Accepted: 15 January 2018

Published online: 15 February 2018

\section{References}

1. Maxson, J. E. et al. Oncogenic CSF3R mutations in chronic neutrophilic leukemia and atypical CML. N. Engl. J. Med. 368, 1781-1790 (2013).

2. Elliott, M. A. et al. ASXL1 mutations are frequent and prognostically detrimental in CSF3R-mutated chronic neutrophilic leukemia. Am. J. Hematol. 90 , 653-656 (2015).

3. Cui, Y. J. et al. The clinical characteristics, gene mutations and prognosis of chronic neutrophilic leukemia. Zhonghua Xue Ye Xue Za Zhi 38, 28-32 (2017).

4. Arber, D. A. et al. The 2016 revision to the World Health Organization classification of myeloid neoplasms and acute leukemia. Blood 127, 2391-2405 (2016). 
5. Pardanani, A. et al. CSF3R T618l is a highly prevalent and specific mutation in chronic neutrophilic leukemia. Leukemia 27, 1870-1873 (2013).

6. Lasho, T. L. et al. Chronic neutrophilic leukemia with concurrent CSF3R and SETBP1 mutations: single colony clonality studies, in vitro sensitivity to JAK inhibitors and lack of treatment response to ruxolitinib. Leukemia $\mathbf{2 8}$, 1363-1365 (2014).

7. Reilly, J. T. Chronic neutrophilic leukaemia: a distinct clinical entity? Brit J. Haematol. 116, 10-18 (2002)
8. Stahl, M. et al. Clinical response to ruxolitinib in CSF3R T618-mutated chronic neutrophilic leukemia. Ann. Hematol. 95, 1197-1200 (2016).

9. Nooruddin, Z. et al. Changes in allele frequencies of CSF3R and SETBP1 mutations and evidence of clonal evolution in a chronic neutrophilic leukemia patient treated with ruxolitinib. Haematologica 102, e207-e209 (2017).

10. Gunawan, A. S. et al. Ruxolitinib, a potent JAK1/JAK2 inhibitor, induces temporary reductions in the allelic burden of concurrent CSF3R mutations in chronic neutrophilic leukemia. Haematologica 102, e238-e240 (2017). 